\title{
Combining Chemotherapy and Radiation Therapy for Liver Cancer: Is the Solution an Intraarterial Approach?
}

\author{
Jean-Francois H. Geschwind ${ }^{1} \cdot$ Nariman Nezami $^{2}$
}

Received: 26 June 2020/ Accepted: 4 July 2020/Published online: 11 August 2020

(C) Springer Science+Business Media, LLC, part of Springer Nature and the Cardiovascular and Interventional Radiological Society of Europe (CIRSE) 2020

The basic principle of radiation therapy is to induce DNA breaks within cancer cells resulting in cellular injury, and ultimately leading to apoptosis, or cell death. Similarly, conventional chemotherapeutic agents act by preferentially disrupting cancer cell mitosis through DNA damage, ultimately inhibiting cellular division [1, 2]. For years, chemotherapy and radiotherapy have been administered concurrently-with great success in some cancers, especially locally advanced solid tumors where the combined approach leads to better local control and improved survival-in an effort to synergize each other. The concept is rather simple: Chemotherapeutic agents are used as radiosensitizing agents, thereby potentiating the effects of radiation therapy. In order to accomplish this task, the chemotherapeutic agent in question must possess properties and characteristics that allow them to perform such a role. Various agents including antimetabolites, platinum-based agents, taxanes, some molecular targeted agents, and others including topoisomerase I inhibitors (such as irinotecan) do possess such properties by (1) directly increasing the initial radiation damage through their incorporation into DNA, (2) inhibiting cellular repair, (3) accumulating cancer cells in a radiosensitive phase or the converse, i.e., eliminating cells in a radioresistant phase, (4) eliminating hypoxic cells, and/ or (5) inhibiting the accelerated repopulation of tumor

Jean-Francois H. Geschwind

jgeschwind@usaveinclinics.com

1 USA Vein Clinics and Oncology Centers, 304 Wainwright Drive, Northbrook, IL, USA

2 Section of Vascular and Interventional Radiology, Department of Radiology and Radiological Sciences, The Johns Hopkins Hospital, Baltimore, MD, USA cells. However, if the combination of chemotherapy and radiation is beneficial against cancer, it is also clearly detrimental to normal tissues because of its enhanced toxicity, which has limited its use in cancers located within organs-liver cancer for example-that are especially vulnerable and sensitive to injury. As a result, combining chemotherapy with radiation is only helpful if the therapeutic benefits on tumors far exceed toxicities on normal tissues [2]. This is the main reason why liver cancer has traditionally been off limits for combined therapy.

By combining chemotherapy and radiation in the form of irinotecan-loaded beads and yttrium-90 glass beads delivered intraarterially within minutes of each other, the authors of the manuscript in the current issue of CVIR demonstrate, in an animal model of metastatic liver cancer, that such combination is not only feasible technically and more potent than either one alone, but also safe [3]. Precedence exists in cervical cancer where chemotherapy infused intraarterially was combined with external radiation therapy to deliver a more potent therapy to target tissues [4]. The novelty here is that both therapies were introduced intraarterially capitalizing on the facts that (1) yttrium-90 as a beta emitter has a low penetration in tissue, thereby preserving healthy liver tissue which is in turn essential to metabolize irinotecan into its active form SN38, and (2) the glass beads used to deliver yttrium-90 to the liver tumors are largely non-embolic and therefore do not restrict arterial inflow preserving oxygenation within the tumor, which, as mentioned above, is a critical element of radiation effect $[3,5]$. This two-prong assault on the liver tumor was made possible by the presence of SN38 permeating the liver tissue which at least in theory was able to reach the cancer cells not reached by the yttrium-90 beads, because of arterial inflow limitation. In other words, 
whatever cancer cells were not destroyed by the yttrium- 90 because of reduced oxygenation at the periphery of the tumor, the SN38 from irinotecan managed to destroy: a perfect example of synergy [6].

One of the key messages of this CVIR manuscript is that the combination of radiation and chemotherapy to treat liver cancer may be possible clinically when administered intraarterially and carefully as described in the study. This was done by using non-embolic particles to deliver the radiation in order to minimize disruption of the tumor microenvironment and toxicity on healthy liver tissue so that the chemotherapeutic agent can do its job. This encouraging study now necessitates a rigorous clinical trial to confirm these exciting findings.

\section{Compliance with Ethical Standards}

Conflict of interest The authors declare that they have no conflict of interest.

\section{References}

1. Memon K, Lewandowski RJ, Kulik L, Riaz A, Mulcahy MF, Salem R. Radioembolization for primary and metastatic liver cancer. Semin Radiat Oncol. 2011;21(4):294-302. https://doi.org/ 10.1016/j.semradonc.2011.05.004.

2. Nishimura Y. Rationale for chemoradiotherapy. Int J Clin Oncol. 2004;9(6):414-20. https://doi.org/10.1007/s10147-004-0443-z.

3. Gordon AC, White SB, Yang Y, et al. Feasibility of combination intra-arterial yttrium-90 and irinotecan microspheres in the VX2 rabbit model. Cardiovasc Intervent Radiol. 2020. https://doi.org/ 10.1007/s00270-020-02538-x.

4. Kokubo M, Tsutsui K, Nagata Y, Okajima K, Katakura Y, Negoro $\mathrm{Y}$, et al. Radiotherapy combined with transcatheter arterial infusion chemotherapy for locally advanced cervical cancer. Acta Oncol. 1998;37(2):143-9. https://doi.org/10.1080/ 028418698429694.

5. Benson AB, Venook AP, Al-Hawary MM, Cederquist L, Chen YJ, Ciombor KK, et al. NCCN guidelines insights: colon cancer, version 2.2018. J Natl Compr Canc Netw. 2018;16(4):359-69. https://doi.org/10.6004/jncen.2018.0021.

6. Graham K, Unger E. Overcoming tumor hypoxia as a barrier to radiotherapy, chemotherapy and immunotherapy in cancer treatment. Int J Nanomed. 2018;13:6049-58. https://doi.org/10.2147/ IJN.S140462.

Publisher's Note Springer Nature remains neutral with regard to jurisdictional claims in published maps and institutional affiliations. 